\title{
Predictive Value of Race and Gender on Gang Attitudes Among Young Adults
}

\author{
Moneque Walker-Pickett ${ }^{1}$, Katrina A. Homer-Darg ${ }^{2}$ \\ ${ }^{1}$ Department of Criminal Justice, Saint Leo University, Saint Leo, USA \\ ${ }^{2}$ Department of Criminal Justice, American Inter Continental University, Schaumburg, USA
}

Email address:

Moneque.Walker-Pickett@SaintLeo.edu (M. Walker-Pickett), KatrinaHomer@Yahoo.com (K. A. Homer-Darg)

To cite this article:

Moneque Walker-Pickett, Katrina A. Homer-Darg. Predictive Value of Race and Gender on Gang Attitudes Among Young Adults. International Journal of Education, Culture and Society. Vol. 2, No. 6, 2017, pp. 203-207. doi: 10.11648/j.ijecs.20170206.17

Received: August 25, 2017; Accepted: October 16, 2017; Published: December 22, 2017

\begin{abstract}
While much research has been done on gangs in general, little research exists that has examined the predictors of attitudes toward gangs held by young adult women in micropolitan communities. The researchers conducted a predictive correlational research study to determine the significant predictors of attitudes toward gangs held by small-metropolitan community young adults between the ages of eighteen and 25. Through survey sampling, the researchers found that gender was a significant predictor of attitudes toward gangs within this sample population, and race was also a significant predictor of attitudes. Recommendations for gang prevention and reduction measures are suggested along with recommendations for future research examining attitudes toward gangs.
\end{abstract}

Keywords: Gangs, Gang Research, Gang Studies, Gang Attitudes, Gender, Race, Young Adult, Youth

\section{Introduction}

Gangs are nothing new in the United States, and have been well researched in urban areas. Gangs continue to spread quickly in non-urban areas. In its most recent report on youth and gangs in the National Youth Gang Survey, the Office of Juvenile Justice and Delinquency Prevention reported that in 2012, there were more than 30,700 estimated gangs in the United States [13]. Gang representation in small cities was at $27.1 \%$, and while there was a small downturn in 2012, between 2002 and 2007, the overall proportion of gangs increased by over $40 \%$ in smaller cities [13]. Gang activity continues to be concentrated in cities with larger populations. However, although largely seen as a "city problem," micropolitan and small metropolitan communities do have gang populations even though many did not view gangs as being a problem in their own communities [21]. Because of these persistent misperceptions, gang problems in micropolitan and small metropolitan communities are not well studied by the current gang research. Weisheit and Wells [22] called for additional gang research focusing on small, non-metropolitan communities, citing that current gang research is lacking and incomplete in terms of non- metropolitan gang studies. Similarly, with current statistics indicating gang activity is increasing at larger rates in smaller cities than larger cities, a focus on research within micropolitan communities is overdue.

Gang membership is widespread in some parts of the population, and positive attitudes toward gang activities and membership have enabled gangs to proliferate freely [25]. In fact, it has been long established that positive attitudes toward gangs are central to the power and growth of gangs [17], [19], [20], [22], [24], [25], [27]. However, even benign attitudes have allowed the spread of gang activities, which expand beyond the historical confines of large metropolitan areas and into the relative seclusion of small metropolitan communities [25]. Researchers have posited that higher levels of positive attitudes toward gangs are related to higher incidences of gang membership among large metropolitan community juveniles [24], [25]. Further, research supports the finding that young adults are engaging in gang activity in these larger communities [8], [14]. What is not well known is how these attitudes affect gang membership in nonmetropolitan areas, thus, it is necessary to examine gangs at non-metropolitan level [22], [25].

The purpose of the study was to determine the relationships between attitudes toward gangs held by small 
metropolitan community adults aged 18-25 years, and more specifically, to look at the role of gender and race in these attitudes. Taking a look at attitudes toward gangs in young adult females, this study will help facilitate gang prevention and reduction measures that specifically target small metropolitan community gangs and extend the knowledge base of this population.

\subsection{Gender and Gangs}

Researchers have described relationships between gang membership via race/ethnicity [4], [7], [9], [20] and gender [1], [3], [10], [12], [19] within large metropolitan communities. In one such study, Winfree et al. [25] examined gang-related attitudes of juveniles living in two cities, differing in populations. Winfree et al. [25] determined that youth who were living in larger cities possessed higher levels of pro-gang attitudes and subsequently, they were engaged in gang membership at higher levels than those not living in larger cities. Weisheit and Wells [22] reinforce these conclusions, similarly calling additional research focusing on gangs in non-metropolitan communities, purporting that current gang research involving non-metropolitan communities is meager and incomplete. Finally, gang research in non-metropolitan communities is necessitated to further advocate the need for preventative and intervention policies aimed at the gang epidemic [22], [25].

While men have traditionally held key roles in gang activity, research has shown that females also hold gang membership, but that females are more likely to perform indirect roles in gang activity [3], [17], [16]. Despite these traditional roles, more recent research has determined that direct female involvement in gang activity is on the rise [11], [15]. As a key variable, it is necessary to determine whether the predictive power of gender has a relationship to attitudes toward gangs. However, it is important to examine the effect that race and ethnicity may play in these attitudes as well. For example, previous research has purported the notion that gang culture is centered on race/ethnicity [7], [20]. Racial lines are also integral in the formation of gangs.

\subsection{Attitudes and Gangs}

The research study was focused on whether an individuals' gender serves as a predictor for attitudes toward gangs. Attitudes play a pivotal role in predicting future behavior; attitudinal traits allow researchers to distinguish why individuals either engage or disengage in criminal behavior [23]. Social scientists have defined criminal behavior as based upon the collected beliefs or attitudes held toward a particular behavior by a group or society [5]. Thus, social attitudes and the values held by a society determine normal behavior or what is believed to be criminal.

Young adults are impressionable during the adolescent years, and peer attitudes are important to the relationship process among young adults, as are social influences [6], [14]. Indeed, even gang members themselves have selfreported that some reasons behind gang membership include the need for family, and the appeal held by older gang members [2].

There is ample research supporting the finding that women and men participate in gangs differently. The roles of women tend to be less defined or fixed. While women are more likely to remain on the periphery or participate indirectly in gang activities, men often participate directly in the daily management and activity of the gang. [1], [3], [19]. Gender, then, may be an important predictor variable in analyzing how positive or negative attitudes form toward gangs when looking at small communities. There may be additional implications when looking at gang attitudes within young adults.

There already exists a body of literature exploring gang attitudes among juveniles in large metropolitan communities. Young people living in large metropolitan communities have been found to possess higher reported levels of positive attitudes toward gangs and higher levels of gang membership than those living in non-metropolitan areas. Other writers have pointed toward how higher positive attitudes toward gangs led to an increase in the levels of gang membership [8], [25]. Utilizing the Attitudes toward Gangs Scale (ATGS), the present study addresses the gap in the existing literature by examining the predictive value of gender in attitude toward gangs in non-metropolitan, or micropolitan, communities in the United States.

Despite ample research indicating that gender serves as a predictor of gang membership, determining whether gender serves as a predictor for attitudes toward gangs within young adult populations in micropolitan and small metropolitan communities was unknown [22], [24], [25]. Previous research found that gender and race/ethnicity are related to gang membership, with males being directly involved in gang activities and females taking on indirect gang roles [1], [3], [12], [19].

\subsection{Research Questions}

The researchers endeavored to study whether gender and race have a predictive effect on the attitude toward gangs held by young adults in smaller communities. Accordingly, our questions presented asked whether a significant relationship exists between gender and gang attitudes held by young adults aged 18-25 in small metropolitan communities, and whether a significant relationship exists between race/ethnicity and gang attitudes held by young adults aged 18-25. The following hypotheses were offered:

$H_{1}$ There will be a significant relationship between attitudes toward gangs held by micropolitan and small metropolitan community adults aged $18-25$ years and gender;

$H_{01}$ There will not be a significant relationship between attitudes toward gangs held by micropolitan and small metropolitan community adults aged 18-25 years and gender.

$\mathrm{H}_{2}$ There will be a significant relationship between race/ethnicity and attitudes toward gangs held by micropolitan and small metropolitan community adults aged 18-25 years;

$H_{02}$ There will not be a significant relationship between 
race/ethnicity and attitudes toward gangs held by micropolitan and small metropolitan community adults aged 18-25 years.

\section{Definition of Terms}

The independent variables "Gender" and "Race/Ethnicity" were used to test the research questions. The remaining definitions assist in understanding the demographic and community characteristics.

Gender. As defined by the World Health Organization (WHO) [26], for the purposes of this study, gender is defined as the socially constructed activities, behaviors, roles, and attributes generally assigned to one sex or the other. Sex refers to the biological classification of being male or female, and gender refers to the behavior, roles, and attributes generally ascribed to men or women. As used in this study, the term gender was synonymous with sex, and the respondent could identify as male, female, or transgendered.

Race/ethnicity. Race and ethnicity was categorized to describe groups that individuals identify with within the context of their communities. For purposes of the study, the categories are defined as: American Indian or Alaska Native; Asian; Black or African American; Native Hawaiian or Other Pacific Islander; and, White. Ethnicity is combined with race in the study, and is defined as Hispanic or Latino; and Not Hispanic or Latino.

Young Adult. Young adult refers to an individual who is not considered a juvenile or minor, and is between the ages of 18 and 25.

Micropolitan community. Larger than a small town, micropolitan community is defined as having between 2,500 and 49,999 inhabitants. Determination of the size of a community is based on the United States' Census Bureau's 2009 census survey results [18]. This definition is consistent with both the National Young Gang Survey and the National Gang Threat Assessment.

Small metropolitan community. This type of community is larger than a micropolitan community, and is defined as having from 50,000 to 99,999 inhabitants. Determination of the size of a community is based on the United States' Census Bureau's 2009 census survey results [18], and is consistent with both the National Young Gang Survey and the National Gang Threat Assessment.

Attitudes toward Gangs. In the study, attitudes toward gangs were measured by the Attitudes toward Gangs Scale (ATGS). A high score indicates a positive attitude about gangs while a low score indicates a negative attitude about gangs.

Sampling Method. Participants were solicited from a local community college, gym, a dental office, as well as other locations where young people between the ages of 18-25 were thought to congregate or work. Participation was obtained in one of two ways: completion of a hard-copy survey or completion of an online survey. A total of 116 completed surveys were received, with 85 hard-copy surveys completed and 31 surveys completed online.

\section{Results}

Participants were between the ages of $18-25$, living in a California city with a population range of 2,500 to 99,999 inhabitants (which was established by the participant disclosing his/her primary city of residence). Attitudes toward gangs held by micropolitan and small metropolitan community adults aged 18-25 years was operationally defined as the attitudes possessed by individuals who live in a micropolitan or small metropolitan community (California locales possessing 2,500 to 99,999 inhabitants) and be aged 18 to 25 years. The sample size included a total of 116 participants $(\mathrm{N}=116)$. The demographic statistics of the study's sample are displayed in Table 1, which displays the demographic indicators by age, gender, and race/ethnicity. More than three-quarters of the respondents were between the ages of 18 and 21 years old. The remaining respondents were between the ages of 22-25 ( $\mathrm{N}=28)$. Sixty-nine of the respondents were male, and 47 were female, resulting in a sample pool with a slight overrepresentation of males. Respondents self-reported as white $(44.8 \%)$, Non-white $(15.5 \%)$, and an overall Hispanic ethnicity of $39.7 \%$.

Table 1. Age, Gender, and Race of Participants/Respondents.

\begin{tabular}{llll}
\hline \multirow{2}{*}{ Demographic Characteristics } & $\begin{array}{l}\text { Participants } \\
\text { (N=116) }\end{array}$ & $\begin{array}{l}\text { Percentage (\%) of } \\
\text { Sample }\end{array}$ \\
\hline \multirow{2}{*}{ Age } & Respondents 18-21 Years Old & 88 & $75.9 \%$ \\
& Respondents 22-25 Years Old & 28 & $24.2 \%$ \\
\multirow{2}{*}{ Gender } & Male & 69 & $59.5 \%$ \\
& Female & 47 & $40.5 \%$ \\
& Other (American Indian or Alaska Native, Asian, Hawaiian/Pacific Islander, or Black) & 18 & $15.5 \%$ \\
& White & 52 & $44.8 \%$ \\
& Hispanic & 46 & $39.7 \%$ \\
\hline
\end{tabular}

Most participants (114 out of 116 participants) reported low to moderate levels of positive attitudes toward gangs, scoring $0-4$ on a 10 point scale. Logistic regression was used to provide additional analysis of the data to determine if gender and race held any predictive value in determining the attitude toward gangs held by the respondents, who were comprised of young adults aged 18-25 living in micropolitan and small metropolitan communities. The initial results indicated that gender was highly associated with attitudes toward gangs. Young men were more likely to possess positive attitudes toward gangs than young women (beta= 0.952). This finding was significant at $p$-value $=0.046$. However, when controlling for race, gender no longer presented as a significant predictor of favorable or 
unfavorable attitudes toward gangs. (See Table 2). Being non-white made it $1.5 \mathrm{x}$ more likely that the respondent held favorable attitudes toward gangs. Specifically, results indicated that Hispanic/Latino, Asian, Black or African American, Native Hawaiian or Other Pacific Islander, and American Indian or Alaska Native's had higher instances of positive attitudes toward gangs. Although gender did continue to serve as a predictor of favorable attitudes toward gangs, the finding was not statistically significant. With attitudes predisposing an individual to perform in a preferential manner, and with gang proliferation ever increasing, further evaluation is necessary to determine whether other significant predictors of attitudes toward gangs exist.

Table 2. Logistic Regression Variables in the Equation.

\begin{tabular}{llllllll}
\hline & & B & S. E. & Wald & Df & Sig. \\
\hline \multirow{3}{*}{ Step $1^{\text {a }}$} & Gender & .813 & .447 & 3.305 & 1 & .069 \\
& Race/Ethnicity & 1.572 & .455 & 11.920 & 1 & .255 \\
& Constant & -.363 & .394 & .848 & 1 & .001 & .357 \\
\hline
\end{tabular}

a. Variable(s) entered on step 1: Gender, Race/Ethnicity.

\section{Conclusion}

The current study surveyed the attitudes of adults aged 1825 years from micropolitan and small metropolitan areas. Gender does serve as a significant predictor for attitudes toward gangs, and more specifically, being male predicts a higher favorable attitude toward gangs in young adults. These findings are consistent with previous research that found that gender maintains high predictive relationships in terms of gang membership in metropolitan areas. [1], [3], [4], $[7], \quad[8],[9], \quad[10]$, [19], [20], [25]. However, the intersectionality presented by being a minority male may be more predictive than simply gender alone. When examining the associative power held by the individual independent variables, race/ethnicity were so highly associated with attitudes toward gangs that additional research is suggested to evaluate the favorable attitudes toward gangs reported by minority males in the subject area. A qualitative study would allow the researchers to examine the underlying causes of these favorable attitudes.

By delineating how young groups perceive gangs, it will become possible to present measures that target specific groups that exhibit higher rates of positive attitudes toward gangs [25]. This knowledge will help law enforcement and gang scholar's better address and implement gang prevention and reduction measures specific toward micropolitan and small metropolitan communities and their inhabitants [24], [25].

\section{References}

[1] Archer, L., \& Grascia, A. M. (2006). Girls, gangs and crime: Profile of the young female offender. Journal of Gang Research, 13(2), 37-49.

[2] Blatchford, C. (Producer). (2004). Chuckie, Midget, and Tortilla Flats. (Television Broadcast). Los Angeles, CA: Fox 11 News.

[3] Brown, W. K. (1977). Black female gangs in Philadelphia. International Journal of Offender Therapy and Comparative Criminology. 21: 221-228.

[4] Decker, S. H., Melde, C., \& Pyrooz, D. C. (2013). What do we know about gangs and gang members and where do we go from here? Justice Quarterly, 30 (3), 369-402.

[5] Durkheim, E. (1999). The normal and the pathological. In S. H. Traub and C. B. Little (eds). Theories of Deviance. (pp. 48). Itasca, IL: F. E. Peacock Publishers.

[6] Franzese, R. J., Covey, H. C., \& Menard, S. (2016). Youth gangs. Charles $\mathrm{C}$ Thomas Publisher.

[7] [7] Freng, A. and Esbensen, F. (2007). Race and gang affiliation: An examination of multiple marginality. Justice Quarterly. 24 (4): 600-628.

[8] Howell, J. C. (2015). The History of Street Gangs in the United States: Their Origins and Transformations. Lexington Books.

[9] Lopez, D. A. and Brummett, P. O. (2003). Gang membership and acculturation: ARSMA-II and choloization. Crime \& Delinquency. 49: 627-642.

[10] Mendoza-Denton, N. (2014). Homegirls: Language and cultural practice among Latina youth gangs. John Wiley \& Sons.

[11] National Alliance of Gang Investigators Association. (2005). 2005 National gang threat assessment. Washington DC: Bureau of Justice Assistance.

[12] National Gang Center. National Youth Gang Survey Analysis. Retrieved September 9, 2016 from $\mathrm{http} / / / \mathrm{www}$. nationalgangcenter.gov/Survey-Analysis.

[13] Office of Juvenile Justice and Delinquency Prevention. (2012). Highlights of the 2012 National Youth Gang Survey. OJJDP Fact Sheet. Retrieved September 9, 2016 from http://www.ojjdp.gov/pubs/248025.pdf.

[14] Shelden, R. G., Tracy, S. K., \& Brown, W. B. (2012). Youth gangs in American society. Nelson Education.

[15] Snethen, G., \& VanPuymbroeck, M. (2008). Girls and physical aggression: Causes, trends, and intervention guided by Social Learning Theory. Journal of Aggression and Violent Behavior: 13 (8), 346-354.

[16] Thornberry, T. P., Krohn, M. D., Lizotte, A. J., ChardWierschem. (1993). The Role of Juvenile Gangs in Facilitating Delinquent Behavior. Journal of Research in Crime and Delinquency, 30 (1), 55-87.

[17] Thrasher, F. M. (1927/1963). The Gang: A study 1,313 gangs in Chicago. Chicago: University of Chicago Press. 
[18] United States Census Bureau. (2009). Retrieved on June 10, 2011 from http://www.census.gov/population/www/metroareas/aboutmet ro.html.

[19] Valdez, A., Mikow, J., \& Cepeda, A. (2006). The role of stress, family, coping, ethnic identity, and mother-daughter relationships on substance use among gang affiliated Hispanic females. Journal of Social Work Practice in the Addictions: 6 (4), 31-54.

[20] Vigil, J. D. (2002). A rainbow of gangs: Street cultures in the mega-city. Austin: University of Texas Press.

[21] Violence Prevention Coalition of Greater Los Angeles. (2007, June). Fact Sheet: Gang Violence. Retrieved on May 14, 2010 from http://www.ph.ucla.edu/scipre/pdf/GANG_VIOLENCE.pdf.

[22] Weisheit, R. A. and Wells, L. E. (2001). The perception of gangs as a problem in nonmetropolitan areas. Criminal Justice Review. 26(2): 170-192.
[23] Wiernik, C. (2007). Attitudes, Beliefs, Behavior, and Criminological Research. Conference Papers -American Sociological Association, 1.

[24] Winfree, L. T., Backstrom, T. V., and Mays, G. L. (1994). Social learning theory, self-reported delinquency, and youth gangs: A new twist on a general theory of crime and delinquency. Youth Society. 26: 147-177.

[25] Winfree, L. T., Bernat, F. P., and Esbensen, F. (2001). Hispanic and anglo gang membership in two southwestern cities. The Social Science Journal. 38: 105-117.

[26] World Health Organization (WHO). (2011). Gender, Women and Health. Retrieved on October 16, 2011 from http://www.who.int/gender/whatisgender/en/.

[27] Zatz, M. S. and Portillos, E. L. (2000). Voices from the barrio: Chicano/a gangs, families, and communities. Criminology. 38(2): 369-401. 